\title{
Preferences for achromatic horizontal, vertical, and square patterns in zebrafish (Danio rerio)
}

\author{
Lisa A Rimstad ${ }^{1,2}$, Adam Holcombe ${ }^{1}$ ， Alicia Pope ${ }^{1}$, Trevor J Hamilton $^{1,2}$ ， Melike P Schalomon ${ }^{\text {corresp. } 1}$ \\ ${ }^{1}$ Department of Psychology, MacEwan University, Edmonton, Alberta, Canada \\ 2 Neuroscience and Mental Health Institute, University of Alberta, Edmonton, Alberta, Canada \\ Corresponding Author: Melike P Schalomon \\ Email address: schalomonm@macewan.ca
}

The zebrafish (Danio rerio) is gaining popularity as a laboratory organism and is used to model many human diseases. Many behavioural measures of locomotion and cognition have been developed that involve the processing of visual stimuli. However, the innate preference for vertical and horizontal stripes in zebrafish is unknown. We tested the preference of adult zebrafish for three achromatic patterns (vertical stripes, horizontal stripes, and squares) at three different size conditions (1, 5, and $10 \mathrm{~mm})$. Each animal was tested once in a rectangular arena, which had a different pattern of the same size condition on the walls of either half of the arena. We show that zebrafish have differential preferences for patterned stimuli at each of the three size conditions. These results suggest that zebrafish have naïve preferences that should be carefully considered when testing zebrafish in paradigms using visual stimuli. 
1 Preferences for achromatic horizontal, vertical, and square patterns in zebrafish (Danio

2 rerio)

3

4 Lisa A. Rimstad ${ }^{1,2}$, Adam Holcombe ${ }^{1}$, Alicia Pope ${ }^{1}$, Trevor J. Hamilton ${ }^{1,2}$, Melike P.

5 Schalomon $^{1}$

$6 \quad{ }^{1}$ Department of Psychology, MacEwan University, Edmonton, AB, Canada

$7 \quad{ }^{2}$ Neuroscience and Mental Health Institute, University of Alberta, Edmonton, AB, Canada

8

9 Corresponding author:

10 Melike Schalomon ${ }^{1}$

11 7-310S, City Centre Campus, 10700 - 104 Avenue, Edmonton, AB, T5J 4S2, Canada

12 Email address: schalomonm@macewan.ca 


\section{Abstract}

15 The zebrafish (Danio rerio) is gaining popularity as a laboratory organism and is used to model

16 many human diseases. Many behavioural measures of locomotion and cognition have been

17 developed that involve the processing of visual stimuli. However, the innate preference for

18 vertical and horizontal stripes in zebrafish is unknown. We tested the preference of adult

19 zebrafish for three achromatic patterns (vertical stripes, horizontal stripes, and squares) at three

20 different size conditions $(1,5$, and $10 \mathrm{~mm})$. Each animal was tested once in a rectangular arena,

21 which had a different pattern of the same size condition on the walls of either half of the arena.

22 We show that zebrafish have differential preferences for patterned stimuli at each of the three

23 size conditions. These results suggest that zebrafish have naïve preferences that should be

24 carefully considered when testing zebrafish in paradigms using visual stimuli. 

research and is regularly used to investigate the causes and treatments of a range of human disorders (Kalueff et al., 2014). In contrast to other commonly used laboratory vertebrates, zebrafish possess many desirable qualities such as high fecundity, external fertilization, and transparent embryos (Panula et al., 2010). In addition to convenience, many zebrafish genes have human homologues and zebrafish brains have the same basic central nervous system regions as mammalian brains, including a significant overlap in neurotransmitter types and distribution (for review see, Panula et al., 2010; Kalueff et al., 2014; Stewart et al., 2015). There are many procedures for manipulating the zebrafish genome and brain development, as well as a large variety of genetic and molecular techniques for the experimental manipulation of zebrafish 38 (Panula et al., 2010; Stewart et al., 2015).

Reliable paradigms are necessary to detect changes in behaviour that result from genetic mutation or testing of any pharmacological or toxicological substance. There are a variety of existing behavioural tests in adult zebrafish including the T-maze, plus maze, spatial alternation task, light/dark test, novel object recognition test, episodic-like memory test, novel tank diving test, novel approach test, and conditioned place preference tests (Norton \& Bally-Cuif, 2010; Kalueff et al., 2014; Hamilton et al., 2016). Some of these paradigms may require the fish to associate coloured stimuli (blue, red, green, or purple) with food rewards (Williams et al., 2002; Colwill et al., 2005). As suggested by previous research, zebrafish can have preexisting preferences for the colour of experimental stimuli, which can lead to potentially incorrect interpretation of data. For instance, one study suggested that zebrafish show an innate preference for low wavelength colours (blue and purple) compared to higher wavelength colours (red and 
50 green) (Colwill et al., 2005), while another study showed a preference for blue and green

51 environments compared to yellow or red ones (Oliveira et al., 2015). Other researchers have

52 found that zebrafish tend to avoid blue, prefer both red and green equally, and show intermediate

53 preference for yellow dependent on what colour it is paired with (Avdesh et al., 2012). In

54 contrast, zebrafish that were conditioned to coloured food demonstrated a strong preference for

55 red regardless of which colour the fish had been originally conditioned to (Spence \& Smith,

56 2008). Furthermore, zebrafish do not demonstrate naïve preferences for complex multicoloured

57 stimuli (May et al., 2016). These studies reflect the complexity of zebrafish colour preferences

58 and the fact that an understanding of these preferences is necessary prior to the use of

59 behavioural paradigms involving coloured stimuli.

60 A simple alternative to coloured stimuli is the use of achromatic black and white patterns.

61 Innate preferences for achromatic patterns have been tested in various model organisms

62 (Cunningham et al., 2006) but, to date, there have been no studies examining the naïve

63 preference of adult zebrafish for basic achromatic pattern stimuli. Other organisms, such as the

64 honeybee, have innate preferences for patterns that confer an evolutionary advantage (Lehrer et

65 al., 1995). Knowledge about innate pattern preferences in zebrafish would allow the

66 development of paradigms that can be used across different laboratories and fish populations

67 regardless of environmental differences and is necessary to avoid bias in the testing of complex

68 behaviours that use patterned stimuli (Bilotta et al., 2005; Tierney, 2011). In this study we tested

69 zebrafish preference for black and white horizontal, vertical, or square patterns at three different

70 sizes of line thickness ( $1 \mathrm{~mm}, 5 \mathrm{~mm}$, and $10 \mathrm{~mm})$. 
(Big Al's, Edmonton, Canada) $(\mathrm{n}=198)$ and housed in 3-L or 10-L polypropylene tanks (AHAB, Aquatic Ecosystems, Inc. Apopka, FL, USA). All fish were between 1-2 years of age during the study and were housed in the habitat for at least 60 days prior to testing. The aquatic habitat was maintained at a water temperature of $26-28^{\circ} \mathrm{C}$, and a $\mathrm{pH}$ between $7.0-8.0$ as previously described (Holcombe et al., 2013; May et al., 2016). Zebrafish were fed an alternating schedule of either freeze-dried shrimp (Omega One Freeze Dried Mysis Shrimp Nutri-treat, OmegaSea Ltd.,

Germany) or commercial flake fish food (New Life Spectrum Optimum Fresh $\mathrm{H}_{2} \mathrm{O}$ Flakes, New

81 Life International Inc. FL, USA) once per day. Lighting was provided by ceiling-mounted

82 fluorescent light tubes on a 12-hour light/dark cycle (lights on at 8:00 am).

The experimental arena used for testing was lined with solid white, non-reflective corrugated plastic, and measured $54.3 \mathrm{~cm}$ long, $9.5 \mathrm{~cm}$ wide, and $9.5 \mathrm{~cm}$ tall. Clear acrylic glass removable dividers were used to divide the tank into three compartments, two arms measuring $22.5 \mathrm{~cm}$ long, and one center compartment measuring $9.3 \mathrm{~cm}$ long (Fig. 1A). The stimuli used were three different achromatic patterns in three different size conditions for a total of nine different stimuli: horizontal stripes, vertical stripes, and squares, at either $1 \mathrm{~mm}, 5 \mathrm{~mm}$, or $10 \mathrm{~mm}$ in size (Fig. 1B). All nine stimuli were matched for luminosity, with 50\% of each stimulus being black and $50 \%$ being white. Stimuli were printed on waterproof white paper with black and white patterns, and measured to fit the three walls of both arena arms. The center, or start

92 compartment, remained lined with only the white corrugated plastic. The laminated stimulus

93 sheets were attached to the arena arms with Velcro to permit easy switching between trials.

94 Pattern combinations for each trial were chosen using a random number generator and the pattern 95 locations were fully counterbalanced. The test tank was filled to a depth of $5 \mathrm{~cm}$ using water 
96 from the aquatic habitat. Water temperature was maintained between $26-28^{\circ} \mathrm{C}$ for the duration of

97 all trials. Fish behaviour was recorded and analyzed using Ethovision XT (v7, Noldus, USA)

98 motion-tracking software and a camera mounted $1 \mathrm{~m}$ above the arena.

99 Behavioural Testing

100 Prior to each testing session, zebrafish were netted from the aquatic habitat and placed in

101 a holding tank beside the testing apparatus until the start of the experiment (10-60 min). Two

102 different patterns were placed in the two arms of the tank and the dividers were put in place. At

103 the start of each trial a zebrafish was netted and placed in the center compartment. The central

104 acrylic glass dividers were immediately removed and the experimental trial commenced. The

105 duration of time zebrafish spent in each of the three unsegregated compartments for the five

106 minute trial was recorded with Ethovision, as was swimming speed in each compartment. At the

107 completion of each trial, the zebrafish was netted and returned to the home tank. The stimuli in

108 each trial consisted of a pattern of a particular size condition in one arm of the arena, and a

109 different pattern of the same size condition in the other arm. Following the completion of 198

110 trials, the data was exported from Ethovision XT to Microsoft Excel and then analyzed using

111 SPSS software. There were 66 zebrafish tested in each of the $1 \mathrm{~mm}, 5 \mathrm{~mm}$, and $10 \mathrm{~mm}$

112 conditions. Each fish was tested for naïve preference and therefore was only tested once,

113 resulting in each fish being tested for preference in the presence of two stimuli (for a given size).

114 This research was approved by the Grant MacEwan University Animal Research Ethics

115 Board, protocol number 05-12-13 and is in accordance with the Canadian Council for Animal

116 Care (CCAC) guidelines.

117 Statistical Analyses 
119 a patterned stimuli by block (stimuli present during the trial, i.e. horizontal stripes with vertical

120 stripes, horizontal stripes with squares, or vertical stripes with squares) and object (pattern, i.e.

121 horizontal stripes, vertical stripes, or squares) design. Object (pattern), block (stimuli

122 combination), and the object by block interaction were treated as fixed effects. The object by

123 block interaction was analyzed to determine whether there was a specific preference for a pattern

124 (ex. vertical stripes) that was more pronounced in a given trial (ex. vertical stripes vs. squares)

125 than the other comparison trial (ex. vertical stripes vs. horizontal stripes). Pairwise comparisons

126 between patterns were made with a Bonferroni adjustment for multiple comparisons. Eleven

127 trials were omitted from the statistical analysis on the basis of tracking errors by Ethovision

128 software $(n=6)$ or because the zebrafish failed to explore both sides of the test arena $(n=5)$.

129 Tracking errors were defined as an inability of the software system to recognize the fish relative

130 to the background picture as a result of the fish being too small or low in contrast relative to the

131 background. The final number of successful trials was 63 in the $1 \mathrm{~mm}$ condition, 64 in the $5 \mathrm{~mm}$

132 condition, and 60 in the $10 \mathrm{~mm}$ condition.

133

134

135

136

137

139

140

\section{Results}

We analyzed the data for effects of stimulus combination within each of the three size conditions by analyzing the amount of time spent in the portion of the arena lined with that particular pattern, as well as velocity $(\mathrm{cm} / \mathrm{s})$ of the fish. For the $10 \mathrm{~mm}$ size condition, both the main effect for object $(F(2,114)=9.11, p<0.001)$, as well as the interaction of block by object $(F(1,114)=9.21, p=0.003)$ were significant (Fig. 2A and Fig. S1A). Zebrafish spent significantly more time in the compartment with vertical stripes $(164 \mathrm{sec}$; SE $=9.94)$ than in compartments with horizontal stripes $(120 \mathrm{sec} ; \mathrm{SE}=9.69)$ or squares $(119 \mathrm{sec} ; \mathrm{SE}=9.82)$. The 
141 interaction effect reflects the increased preference for the vertical stripe pattern when it was

142 paired with the square pattern relative to when the vertical stripe pattern was paired with the

143 horizontal stripe pattern. There was no significant main effect for block $(F(2,114)=2.58, p=$

144 0.08). The velocity analysis showed no significant main effects for block $(F(2,114)=0.64, p=$

$1450.53)$ or object $(F(2,114)=0.15, p=0.86$; Fig. $2 \mathrm{~B}$, and Fig. S1B $)$. There was also no significant

146 block by object interaction effect $(F(2,114)=0.047, p=0.83$. The swim speed of the zebrafish

147 was not significantly different in response to any of the three patterns at this size (horizontal

148 stripes: $9.41 \mathrm{~cm} / \mathrm{s}$; vertical stripes: $8.70 \mathrm{~cm} / \mathrm{s}$; squares: $9.30 \mathrm{~cm} / \mathrm{s}$ ).

149 For the $5 \mathrm{~mm}$ size condition, both the main effect for block $(F(2,122)=25.8, p<0.001)$

150 and the main effect for object $(F(2,122)=95.2, p<0.001)$ were significant (Fig. 3A and Fig.

151 S2A). Zebrafish spent significantly less time in the compartment with squares $(69.4 \mathrm{sec} ; \mathrm{SE}=$

152 6.24), whether it was paired with a compartment with vertical stripes $(159 \mathrm{sec} ; \mathrm{SE}=6.31)$ or

153 with horizontal stripes $(160 \mathrm{sec} ; \mathrm{SE}=6.24)$. There was no significant effect for the interaction of

154 block by object $(F(2,122)=0.015, p=0.90)$. In the velocity analysis, there were significant

155 main effects for both block $(F(2,122)=3.91, p=0.023)$ and for object $(F(2,122)=13.2, p<$

156 0.001; Fig. 3B and Fig. S2B). Zebrafish swam significantly faster in the compartment with

157 squares $(9.75 \mathrm{~cm} / \mathrm{s} ; \mathrm{SE}=0.29)$ whether the pattern was paired with vertical stripes $(8.44 \mathrm{~cm} / \mathrm{s}$;

$158 \mathrm{SE}=0.29)$ or horizontal stripes $(8.05 \mathrm{~cm} / \mathrm{s} ; \mathrm{SE}=0.29)$. There was no significant block by object

159 interaction effect $(F(2,122)=0.026, p=0.87)$.

160 For the $1 \mathrm{~mm}$ size condition, both the main effect for block $(F(2,120)=5.39, p=0.006)$

161 and the main effect for object $(F(2,120)=19.3, p<0.001)$ were significant (Fig. 4A and Fig.

162 S3A). Zebrafish spent significantly more time in the compartment with horizontal stripes (155

$163 \mathrm{sec} ; \mathrm{SE}=6.60)$ regardless of whether it was paired with a compartment with vertical stripes (117 
$164 \mathrm{sec} ; \mathrm{SE}=6.67)$ or with squares $(109 \mathrm{sec} ; \mathrm{SE}=6.51)$. There was no significant effect for the

165 interaction of block by object $(F(2,120)=2.31, p=0.13)$. The velocity analysis showed a

166 significant main effect for object $(F(2,120)=3.19, p=0.045$; Fig. 4B and Fig. S3B). Overall,

167 zebrafish swam significantly slower in the compartment with horizontal stripes $(7.48 \mathrm{~cm} / \mathrm{s} ; \mathrm{SE}=$

168 0.32), as compared to the compartments with vertical stripes $(9.07 \mathrm{~cm} / \mathrm{s} ; \mathrm{SE}=0.32)$ or with the

169 square pattern $(9.04 \mathrm{~cm} / \mathrm{s} ; \mathrm{SE}=0.31)$. There was no significant main effect for block $(F(2,120)$

$170=2.12, p=0.12)$ or for the block by object interaction $(F(2,120)=0.58, p=0.45)$.

\section{Discussion}

172

Zebrafish show pattern preferences with horizontal, vertical, and square patterns at size

conditions of $1 \mathrm{~mm}, 5 \mathrm{~mm}$, and $10 \mathrm{~mm}$, demonstrating that zebrafish have the ability to

174 discriminate between patterns of such sizes. Vertical lines were significantly preferred at a size

175 condition of $10 \mathrm{~mm}$ and horizontal lines at a size condition of $1 \mathrm{~mm}$. At the intermediate size

176 condition of $5 \mathrm{~mm}$ the subjects preferred neither vertical nor horizontal lines but did show a

177 significant avoidance of the square pattern. The zebrafish also showed an increase in swim speed

178 in response to the non-preferred $5 \mathrm{~mm}$ square pattern, as well as a decrease in swim speed in

179 response to the preferred $1 \mathrm{~mm}$ horizontal pattern. At the $10 \mathrm{~mm}$ size condition there was no

180 difference in swim speed in response to any of the three patterns.

181 In their natural habitat, zebrafish use vegetation as shelter from predators as well as for

182 concealment during spawning and foraging (Spence et al., 2007; Spence et al., 2008). Zebrafish

183 have been shown to prefer environments that include vegetation in the laboratory and display

184 less aggressive behaviours when vegetation is present (Basquill \& Grant, 1998; Engeszer et al.,

185 2007b; Spence et al., 2008). Additionally, vegetation can ease the process of inflating the swim

186 bladder in larval zebrafish, possibly conferring a survival advantage on fish spawning in 
187 vegetated areas (Spence et al., 2007; Spence et al., 2008). The grasses present in a natural

188 zebrafish environment may be visually similar to the $10 \mathrm{~mm}$ vertical lines preferred in this 189 experiment.

190 It is plausible that the $5 \mathrm{~mm}$ square pattern is aversive to zebrafish and that horizontal and 191 vertical lines of this size are both neutral stimuli. Additional evidence for the aversive nature of

192 the $5 \mathrm{~mm}$ square pattern comes from the increase in swim speed, an indicator of anxiety (Kalueff 193 et al., 2013), which occurs relative to the other patterns at this size condition. It is possible that 194 an innate avoidance of this pattern is related to the spotted appearance of common predatory 195 species that zebrafish encounter in their natural environment, such as the blotched snakehead 196 (Channa maculata), northern snakehead (Channa argus), or Gangetic leaffish (Nandus nandus)

197 (Engeszer et al., 2007b; Gerlai, 2013).

198 The preference for horizontal lines of $1 \mathrm{~mm}$ may be explained by the horizontal stripes of 199 a similar width that characterize the appearance of conspecifics. This would be consistent with 200 the lack of horizontal preference at thicker line widths. Zebrafish tend to form a preference for 201 stripes that is reliant on early learning experiences during a critical period that result from an 202 imprinting-like phenomenon (Engeszer et al., 2004; Rosenthal \& Ryan, 2005; Engeszer et al., 203 2007a). The zebrafish used in this study were likely housed with wild-type (striped) fish from 204 hatching and are therefore likely to express a strong preference for the $1 \mathrm{~mm}$ wide horizontal 205 stripe patterns characteristic of conspecifics. Zebrafish stripes play a significant role in shoaling 206 behaviour, as well as in the mediation of aggressive and mating behaviours between conspecifics 207 (Rosenthal \& Ryan, 2005; Engeszer et al., 2007a). Therefore, zebrafish that were raised with 208 phenotypically similar tank mates can be expected to show a preference for a $1 \mathrm{~mm}$ horizontal 209 striped pattern. In addition to a preference for the horizontal pattern, the zebrafish in this study 
210 also showed a decrease in swim speed in the compartment with the horizontal stripes at the $1 \mathrm{~mm}$

211 size but not at $5 \mathrm{~mm}$ or $10 \mathrm{~mm}$. High swim speed is indicative of anxiety in zebrafish (Kalueff et

212 al., 2013), thus the decrease in swim speed in response to the horizontal stripe pattern may

213 indicate a decreased stress response. To date there have been many studies on location

214 preference of zebrafish in the presence of various colour stimuli (Avdesh et al., 2012; Bault et

215 al., 2015; Olivera et al., 2015) but few have examined mobility within the stimulus compartment

216 in a two choice test. However, when the walls of an arena are either black, white, or transparent

217 there is a significant effect on immobility which increases with transparent walls (Blaser \&

218 Rosemberg, 2012). Indeed, the duration of time spent in a zone containing a stimulus is

219 informative, but so is the level of movement within that compartment itself, which is why the

220 speed (or immobility) of the fish may also be of interest. Future studies with anxiolytic or 221 anxiogenic drugs could investigate this in more detail.

222 This experiment demonstrates that adult zebrafish have the ability to differentiate

223 between achromatic patterns ranging from $1 \mathrm{~mm}$ to $10 \mathrm{~mm}$ in size. Knowledge of visual acuity in

224 zebrafish is relevant for behavioural paradigms that rely on the perceptual abilities of zebrafish,

225 such as ones that use patterned test tanks or object discrimination tasks. The results in this study

226 suggest that zebrafish have naïve preferences for some patterns in their surroundings, and they

227 suggest that the visual acuity of zebrafish permit them to perceive patterns with a resolution of 1

$228 \mathrm{~mm}$. There were no relative preferences for the $10 \mathrm{~mm}$ horizontal lines or squares, the $5 \mathrm{~mm}$

229 horizontal or vertical lines, and the $1 \mathrm{~mm}$ squares and vertical lines, suggesting that these

230 patterns would be suitable for use in place preference paradigms. The significance of the

231 preferences for the pattern sizes is further substantiated by the fact that the zebrafish used in this

232 study had diverse experimental and genetic backgrounds. However, as this is the first study to 
233 examine preferences for patterned environments in zebrafish, more testing would be beneficial to

234 confirm the results across multiple strains. Additionally, future studies may benefit from

235 analyzing pattern preferences results by sex, as recent studies suggest zebrafish behaviour varies

236 between males and females (Tran \& Gerlai 2013; Lucon-Xiccato \& Bisazza, 2017). As zebrafish

237 continue to gain popularity as a model organism in the study of vertebrate disease, the

238 development of reliable behavioural paradigms will be increasingly crucial. Knowing which

239 visual stimuli are preferred or avoided by naïve subjects is key in the development of these

240 paradigms in order to reduce the possibility of biased or invalid data.

241

242

Acknowledgements

243 We would like to thank Karen Buro (MacEwan University) for her assistance with the

244 statistical analysis. We would also like to thank Jay Abbott (Animal Care Technician, MacEwan

245 University) for his assistance with animal husbandry. 
248

249

250

251

252

253

254

255

256

257

258

259

260

261

262

263

264

265

266

267

268

269

270

271

272

273

274

275

276

277

278

279

280

281

282

283

284

285

286

287

288

289

290

Avdesh A, Martin-Iverson MT, Mondal A, Chen MQ, Askraba S, Morgan N, Lardelli M, Groth DM, Verdile G, Martins RN. 2012. Evaluation of color preference in zebrafish for learning and memory. Journal of Alzheimers Disease 28:459-469. DOI:10.3233/Jad2011-110704

Basquill SP, Grant JWA. 1998. An increase in habitat complexity reduces aggression and monopolization of food by zebra fish (Danio rerio). Canadian Journal of Zoology-Revue Canadienne De Zoologie 76:770-772. DOI: 10.1139/cjz-76-4-770

Bault ZA, Peterson SM, Freeman JL. 2015. Directional and color preference in adult zebrafish: Implications in behavioral and learning assays in neurotoxicology studies. Journal of Applied Toxicology 35:1502-1510. DOI: 10.1002/jat.3169

Bilotta J, Risner ML, Davis EC, Haggbloom SJ. 2005. Assessing appetitive choice discrimination learning in zebrafish. Zebrafish 2:259-268. DOI: 10.1089/zeb.2005.2.259

Blaser RE, Rosemberg DB. 2012. Measures of anxiety in zebrafish (Danio rerio): dissociation of black/white preference and novel tank test. PLoS One 7:e36931. DOI:

10.1371/journal.pone.0036931

Colwill RM, Raymond MP, Ferreira L, Escudero H. 2005. Visual discrimination learning in zebrafish (Danio rerio). Behavioural Processes 70:19-31. DOI:

10.1016/j.beproc.2005.03.001

Cunningham CL, Gremel CM, Groblewski PA. 2006. Drug-induced conditioned place preference and aversion in mice. Nature Protocols 1:1662-1670. DOI: 10.1038/nprot.2006.279

Engeszer RE, Alberici da Barbiano L, Ryan MJ, Parichy DM. 2007a. Timing and plasticity of shoaling behaviour in the zebrafish, Danio rerio. Animal Behaviour 74:1269-1275. DOI: 10.1016/j.anbehav.2007.01.032

Engeszer RE, Patterson LB, Rao AA, Parichy DM. 2007b. Zebrafish in the wild: A review of natural history and new notes from the field. Zebrafish 4:21-U126. DOI: 10.1089/zeb.2006.9997

Engeszer RE, Ryan MJ, Parichy DM. 2004. Learned social preference in zebrafish. Current Biology 14:881-884. DOI: 10.1016/j.cub.2004.04.042

Gerlai R. 2013. Antipredatory behavior of zebrafish: adaptive function and a tool for translational research. Evolutionary Psychology 11:591-605.

Hamilton TJ, Myggland A, Duperreault E, May Z, Gallup J, Powell RA, Schalomon M, Digweed SM. 2016. Episodic-like memory in zebrafish. Animal Cognition 19:1071-1079. DOI: 10.1007/s10071-016-1014-1

Holcombe A, Howorko A, Powell RA, Schalomon M, Hamilton TJ. 2013. Reversed scototaxis during withdrawal after daily-moderate, but not weekly-binge, administration of ethanol in zebrafish. PLoS One 8:e63319. DOI: 10.1371/journal.pone.0063319

Kalueff AV, Gebhardt M, Stewart AM, Cachat JM, Brimmer M, Chawla JS, Craddock C, Kyzar EJ, Roth A, Landsman S, Gaikwad S, Robinson K, Baatrup E, Tierney K, Shamchuk A, Norton W, Miller N, Nicolson T, Braubach O, Gilman CP, Pittman J, Rosemberg DB, Gerlai R, Echevarria D, Lamb E, Neuhauss SC, Weng W, Bally-Cuif L, Schneider H, Zebrafish Neuroscience Research C. 2013. Towards a comprehensive catalog of zebrafish behavior 1.0 and beyond. Zebrafish 10:70-86. DOI: 10.1089/zeb.2012.0861 
291

292

293

294

295

296

297

298

299

300

301

302

303

304

305

306

307

308

309

310

311

312

313

314

315

316

317

318

319

320

321

322

323

324

325

326

327

328

Kalueff AV, Stewart AM, Gerlai R. 2014. Zebrafish as an emerging model for studying complex brain disorders. Trends in Pharmacological Sciences 35:63-75. DOI: 10.1016/j.tips.2013.12.002

Lehrer M, Horridge GA, Zhang SW, Gadagkar R. 1995. Shape vision in bees: innate preference for flower-like patterns. Philosophical Transactions: Biological Sciences 347:15.

Lucon-Xiccato T, Bisazza A. 2017. Individual differences in cognition among teleost fishes. Behavioral Processes 141:184-195. DOI: 10.1016/j.beproc.2017.05.015

May Z, Morrill A, Holcombe A, Johnston T, Gallup J, Fouad K, Schalomon M, Hamilton TJ. 2016. Object recognition memory in zebrafish. Behavioural Brain Research 296:199210. DOI: $10.1016 /$ j.bbr.2015.09.016

Norton W, Bally-Cuif L. 2010. Adult zebrafish as a model organism for behavioural genetics. BMC Neuroscience 11:90. DOI: 10.1186/1471-2202-11-90

Oliveira J, Silveira M, Chacon D, Luchiari A. 2015. The zebrafish world of colors and shapes: Preference and discrimination. Zebrafish 12:166-173. DOI: 10.1089/zeb.2014.1019

Panula P, Chen YC, Priyadarshini M, Kudo H, Semenova S, Sundvik M, Sallinen V. 2010. The comparative neuroanatomy and neurochemistry of zebrafish CNS systems of relevance to human neuropsychiatric diseases. Neurobiology of Disease 40:46-57. DOI: 10.1016/j.nbd.2010.05.010

Rosenthal GG, Ryan MJ. 2005. Assortative preferences for stripes in danios. Animal Behaviour 70:1063-1066. DOI: 10.1016/j.anbehav.2005.02.005

Spence R, Ashton R, Smith C. 2007. Oviposition decisions are mediated by spawning site quality in wild and domesticated zebrafish, Danio rerio. Behaviour 144:953-966. DOI: $10.1163 / 156853907781492726$

Spence R, Gerlach G, Lawrence C, Smith C. 2008. The behaviour and ecology of the zebrafish, Danio rerio. Biological Reviews 83:13-34. DOI: 10.1111/j.1469-185X.2007.00030.x

Spence R, Smith C. 2008. Innate and learned colour preference in the zebrafish, Danio rerio. Ethology 114:582-588. DOI: 10.1111/j.1439-0310.2008.01515.x

Stewart AM, Ullmann JF, Norton WH, Parker MO, Brennan CH, Gerlai R, Kalueff AV. 2015. Molecular psychiatry of zebrafish. Molecular Psychiatry 20:2-17. DOI: $10.1038 / \mathrm{mp} .2014 .128$

Tierney KB. 2011. Behavioural assessments of neurotoxic effects and neurodegeneration in zebrafish. Biochimica Et Biophysica Acta-Molecular Basis of Disease 1812:381-389. DOI: 10.1016/j.bbadis.2010.10.011

Tran S., Gerlai R. 2013. Individual differences in zebrafish (Danio rerio). Behavioural Brain Research 257:224-229. DOI: 10.1016/j.bbr.2013.09.040

Williams FE, White D, Messer WS. 2002. A simple spatial alternation task for assessing memory function in zebrafish. Behavioural Processes 58:125-132. DOI: 10.1016/S03766357(02)00025-6 


\section{Figure 1}

Behavioural Testing Apparatus.

A) Schematic of the testing tank. The whole tank (unsegregated) measured $54.3 \mathrm{~cm}$ long $\mathrm{x}$ $9.5 \mathrm{~cm}$ wide $\times 9.5 \mathrm{~cm}$ tall. Clear acrylic glass removable dividers were used to divide the tank into three compartments, two arms $(22.5 \mathrm{~cm}$ long $)$ and one center compartment $(9.3 \mathrm{~cm}$ long) prior to the start of the trial. B) Representative sample of each pattern (horizontal stripes, vertical stripes, and squares) for each size condition (10 mm, $5 \mathrm{~mm}$, and $1 \mathrm{~mm}$ ).

A

B

Removable dividers
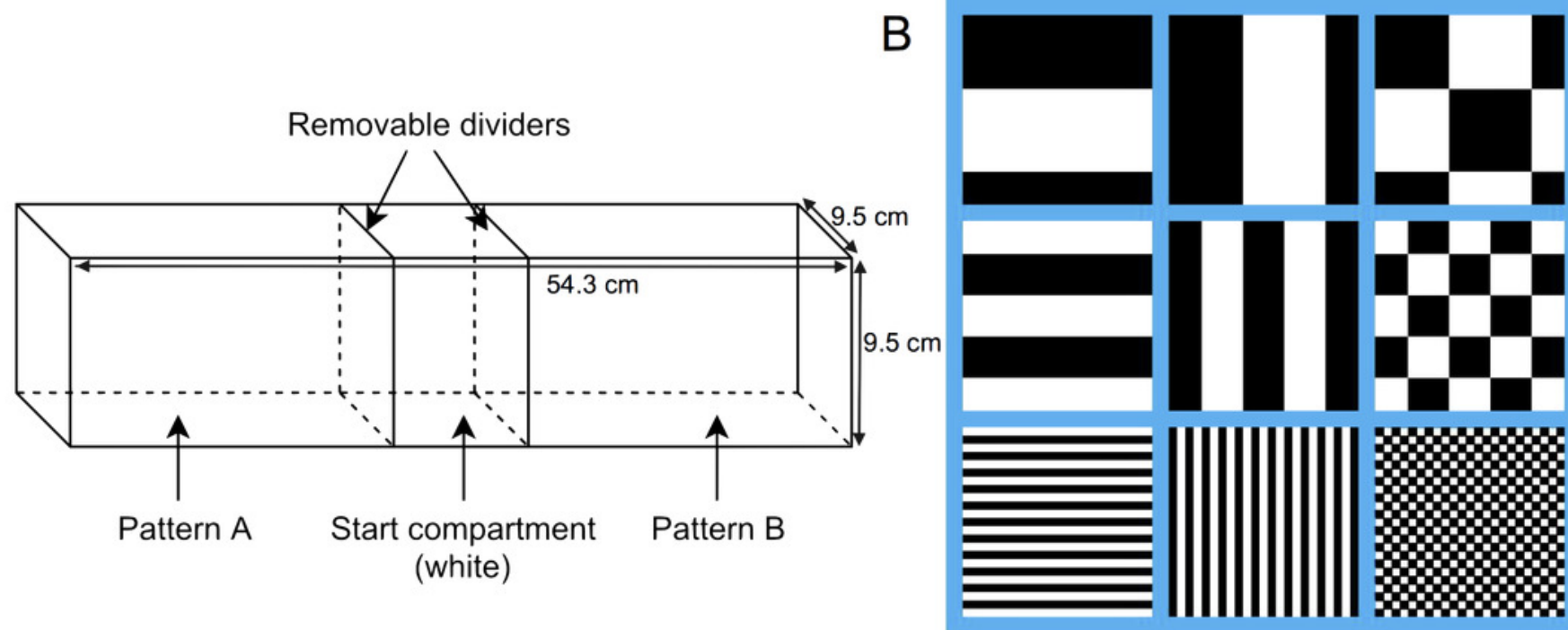


\section{Figure 2 (on next page)}

Time in zone and swim speed for $10 \mathrm{~mm}$ condition.
A) Time spent in the zone with the indicated pattern (mean \pm SEM). Fish spent significantly more time in the zone with the vertical stripes $(p<0.001)$. B) Swim speed as measured by velocity (mean \pm SEM) of the fish within each of the pattern types. The swim speed of the fish did not differ between the three patterns. 


\section{Figure 3 (on next page)}

Time in zone and swim speed for $5 \mathrm{~mm}$ condition.
A) Time spent in the zone with the indicated pattern (mean \pm SEM). Fish spent significantly less time in the zone with the square pattern $(p<0.001)$. B) Swim speed as measured by velocity (mean \pm SEM) of the fish within each of the pattern types. The fish swam significantly faster in the zone with the square pattern $(p<0.001)$. 


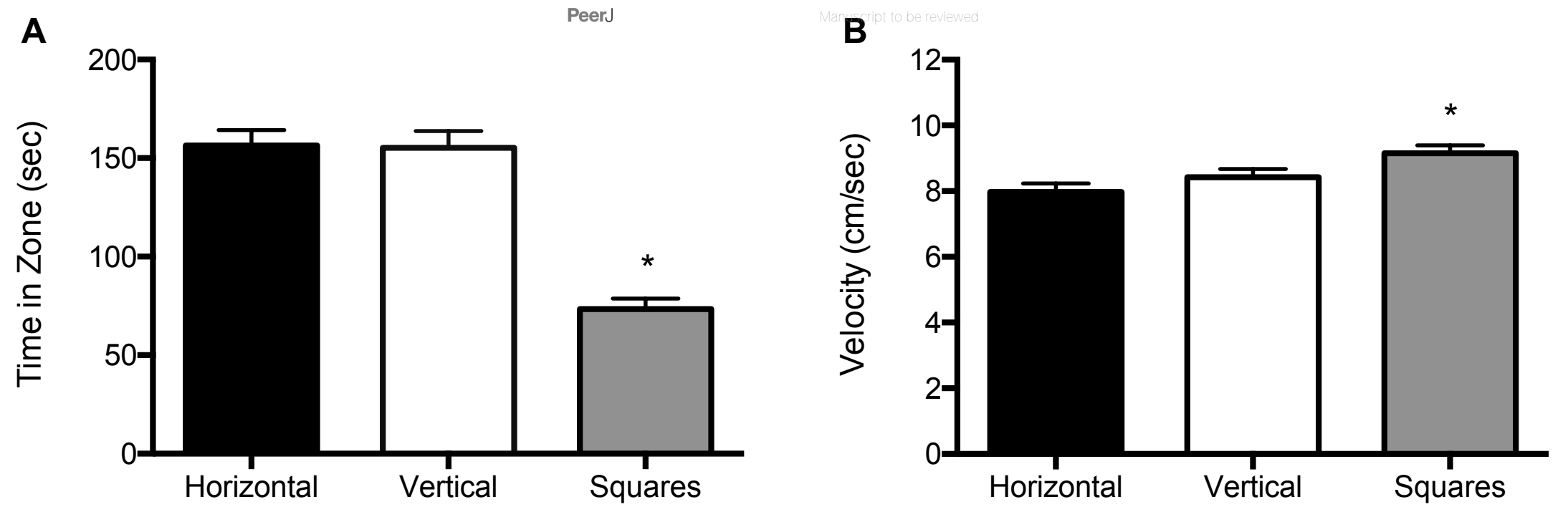




\section{Figure 4 (on next page)}

Time in zone and swim speed for $1 \mathrm{~mm}$ condition.
A) Time spent in the zone with the indicated pattern (mean \pm SEM). Fish spent significantly more time in the zone with the horizontal stripes $(p<0.001)$. B) Swim speed as measured by velocity (mean \pm SEM) of the fish within each of the pattern types. The fish swam significantly slower in the zone with horizontal stripes $(p<0.05)$. 


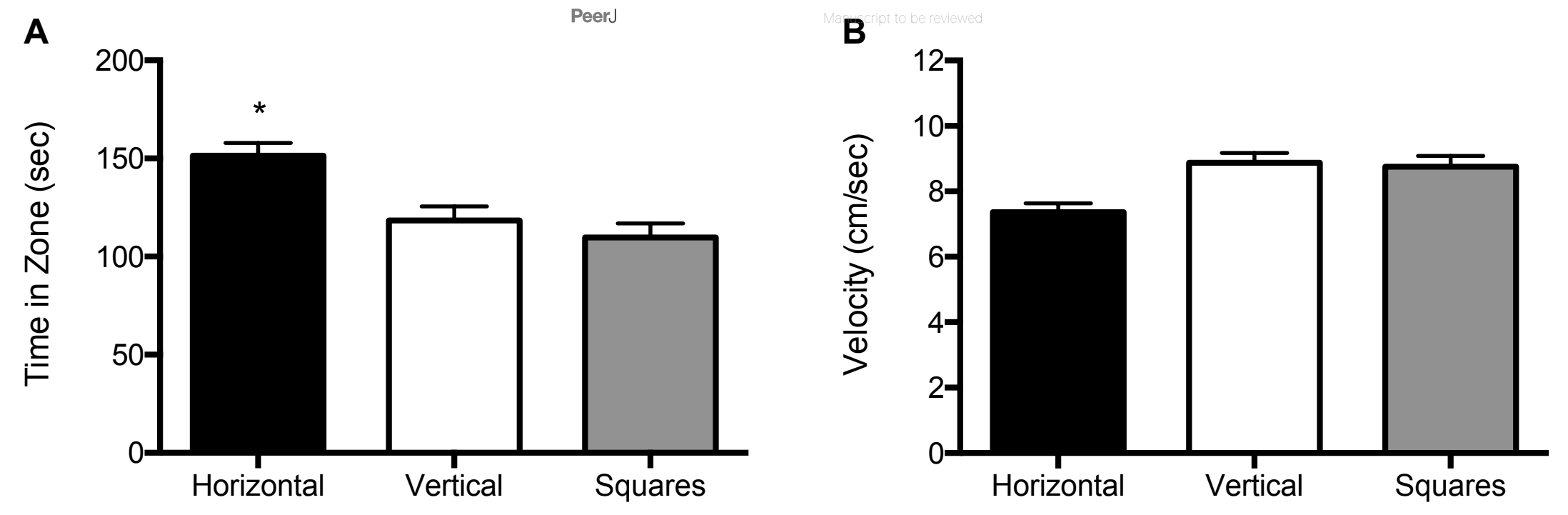

